\title{
Respiratory syncytial virus-associated seizures in Korean children, 2011-2016
}

\author{
Teahyen Cha, MD', Young Jin Choi, MD', Jae-Won Oh, MD, PhD', Chang-Ryul Kim, MD, PhD', Dong Woo Park, MD, PhD², In Joon Seol, \\ $\mathrm{MD}, \mathrm{PhD}{ }^{1}$, Jin-Hwa Moon, MD, PhD' \\ ${ }^{1}$ Department of Pediatrics, Hanyang University College of Medicine, Seoul, Korea \\ ${ }^{2}$ Department of Radiology, Hanyang University Guri Hospital, Hanyang University College of Medicine, Guri, Korea
}

Purpose: Respiratory syncytial virus (RSV) infection can cause various neurological complications. This study aimed to investigate the RSV-associated neurologic manifestations that present with seizures.

Methods: We retrospectively reviewed the medical records of patients aged less than 15 years with laboratory-confirmed RSV infections and seizures between January 2011 and December 2016 in a regional hospital in South Korea.

Results: During this period, 1,193 patients with laboratory-confirmed RSV infection were identified. Of these, 35 ( 35 of 1,193, 2.93\%; boys, 19; girls, 16; mean age: $20.8 \pm 16.6$ months) presented with seizure. Febrile seizure was the most common diagnosis ( 27 of $35,77.1 \%$ ); simple febrile seizures in 13 patients (13 of $27,48.1 \%$ ) and complex febrile seizures in 14 (14 of 27, 51.9\%). Afebrile seizures without meningitis or encephalopathy were observed in 5 patients ( 5 of $35,14.3 \%$ ), seizures with meningitis in 2 ( 2 of 35 , $5.7 \%$ ), and seizure with encephalopathy in 1 (1 of $35,2.9 \%$ ) patient. Lower respiratory symptoms were not observed in 8 patients. In a patient with encephalopathy, brain diffusion-weighted magnetic resonance imaging revealed transient changes in white matter, suggesting cytotoxic edema as the mechanism underlying encephalopathy. Most patients recovered with general management, and progression to epilepsy was noted in only 1 patient.

Conclusion: Although febrile seizures are the most common type of seizure associated with RSV infection, the proportion of patients with complex febrile seizures was higher than that of those with general febrile seizures. Transient cytotoxic edema may be a pathogenic mechanism in RSV-related encephalopathy with seizures.

Key words: Respiratory syncytial virus, Child, Seizure, Magnetic resonance imaging

\section{Introduction}

The respiratory syncytial virus (RSV) is an important cause of acute respiratory tract infection in young children. The common respiratory manifestations are bronchiolitis, pneumonia, bronchitis, and croup. By the age of 2 years, approximately 90\% children are infected by RSV, and approximately $45 \%$ of the RSV-related hospital admissions occur in children younger than 6 months. ${ }^{1,2)}$

As in other common viral infections, RSV may be associated with neurological manifestations, including seizures, encephalopathy, extraocular movement disorder, and central apnea.

${ }^{3-8)}$ Although the pathogenesis of the neurologic complications are not fully understood, the possible contributions of immune-related cytokine responses, less well-proven direct invasion of virus particles, and brain stem-related mechanisms for respiratory control have been recognized. ${ }^{3,5,-11)}$
Corresponding author: Jin-Hwa Moon, MD, PhD Department of Pediatrics, Pediatric Neurology, Hanyang University Guri Hospital, 153 Gyeongchun-ro, Guri 11923, Korea Tel: +82-31-560-2258

Fax: +82-31-552-9493

E-mail: jinhwamoon@hanyang.ac.kr https://orcid.org/0000-0003-0235-5318

Received: 11 September, 2018

Revised: 14 October, 2018

Accepted: 22 October, 2018
This is an open-access article distributed under the terms of the Creative Commons Attribution NonCommercial License (http://creativecommons.org/ licenses/by-nc/4.0/) which permits unrestricted noncommercial use, distribution, and reproduction in any medium, provided the original work is properly cited. 
The incidence of RSV-associated neurologic complications varies across studies and study populations. Sweetman et al. ${ }^{7)}$ reported the incidence of RSV-associated neurologic complications to be $1.2 \%$, which did not include cases with simple febrile seizure, and $\mathrm{Ng}$ et al. ${ }^{12)}$ reported the incidence of RSV-associated encephalopathy to be $1.8 \%$. In a pediatric intensive care unit (PICU)-based study, Kho et al. ${ }^{13)}$ reviewed the cases of children between birth and 2 years of age with acute neurological symptoms and identified 39.1\% of them as RSV-positive. In contrast, Millichap and Wainwright ${ }^{5}$ reported that only $1 \%$ of the RSV-positive patients were admitted to the PICU with neurologic complications. In a study comparing the common viruses responsible for febrile seizure, Chung and Wong ${ }^{14)}$ revealed that the incidence of febrile seizure for RSV was 5.3\%, and among them, complex febrile seizure was observed in 13.6\% of cases, which was lower than the percentage of such cases for influenza and adenovirus.

In a previous study in Korea during November 2002 and June 2007, Yoon et al. ${ }^{15)}$ reported that the overall incidences of RSVassociated neurologic complications and seizures were $7.1 \%$ and $1.9 \%$, respectively. Another study in Korea by Park et al. ${ }^{16)}$ reported that the incidence of RSV-associated encephalitis was $0.08 \%$. The same study showed that RSV-associated brain magnetic resonance imaging (MRI) findings may resemble those of other viral and limbic encephalitis, and no abnormality on diffusion-weighted imaging (DWI) was found. ${ }^{16,17)}$

Although the study by Yoon et al. ${ }^{15)}$ was the first and the only study on the overall RSV-associated neurologic complications in Korea, further analysis of patients with seizure based on presumed causes was needed. In addition, most of the previous studies were regarding encephalopathy or relatively severe patients, and did not focus on overall features of RSV-associated seizures. It has not yet been fully determined whether the clinical characteristics of the RSV-associated febrile seizure are similar to those of the general febrile seizure, and whether RSV-associated afebrile seizures are benign situation-related seizures, as reported by Miyama and Goto. ${ }^{6}$ Based on this background, we aimed to investigate the RSVassociated neurologic manifestations in children who presented with seizures. Furthermore, we report a transient DWI change in a young child with acute encephalopathy symptoms exhibiting seizures.

\section{Materials and methods}

\section{Patients}

The medical records of the patients admitted to Hanyang University Guri Hospital from January 2011 to December 2016 were reviewed retrospectively. The inclusion criteria were as follows: children younger than 15 years, laboratory-confirmed RSV infection, and presentation of neurologic symptoms with seizures during the infection. The patients whose neurological symptoms were caused by factors other than RSV infections, and those with epilepsy were excluded. In this study, a simple febrile seizure was defined as a seizure occurring during a febrile illness and not caused by an acute disease of the nervous system in children aged between 6 months and 5 years. ${ }^{18)}$ The seizure should be generalized and short (duration less than 15 minutes) and not recur within 24 hours. Complex febrile seizure was defined as a 1) focal, 2) generalized, and prolonged seizure lasting more than 15 minutes and recurring with 24 hours, or 3) postictal neurological abnormalities, such as Todd's palsy. ${ }^{18)}$ This study was performed in a regional hospital in Guri, a city located in the metropolitan area around Seoul. All research protocols were approved by the Institutional Review Board (IRB) at Hanyang University Guri Hospital (approval number: 2017-12-014). Informed consent was exempted by the IRB.

\section{Data collection}

Demographic information and clinical courses of the patients; information regarding combined diseases; results of blood tests; cerebrospinal fluid (CSF) examinations; electroencephalography (EEG); and brain imaging techniques such as MRI (including DWI) were analyzed. Detection of RSV was performed using real-time reverse transcription-polymerase chain reaction, with samples obtained by nasopharyngeal swabbing (AdvanSure, LG, Seoul, Korea).

\section{Statistical analysis}

Descriptive statistics were used to analyze demographic data. The continuous variables are presented as mean \pm standard deviation. The statistical analysis was performed using IBM SPSS Statistics ver. 21.0 (IBM Co., Armonk, NY, USA). The incidence reported by national RSV surveillance during the study period was used for comparison with the incidence pattern of this study.

\section{Results}

\section{Demographic data}

We identified a total of 1,193 children who were diagnosed with laboratory-confirmed RSV infection during the study period; 35

Table 1. Clinical manifestations of children with respiratory syncytial virus-associated seizures

\begin{tabular}{lccc}
\hline Manifestation & No. $(\%)$ & Age $(\mathrm{mo})$, mean \pm SD & Sex, boy:girl \\
\hline Febrile seizure only & & & \\
$\quad$ Simple & $13(37.1)$ & $24.3 \pm 13.1$ & $6: 7$ \\
$\quad$ Complex & $14(40.0)$ & $22.6 \pm 20.1$ & $9: 5$ \\
Afebrile seizure only & $5(14.3)$ & $16.8 \pm 14.4$ & $4: 1$ \\
Encephalopathy with seizure & $1(2.9)$ & 1.6 & $0: 1$ \\
Meningitis with seizure & $2(5.7)$ & $4.0 \pm 4.2$ & $0: 2$ \\
Total & $35(100)$ & $20.8 \pm 16.6$ & $19: 16$
\end{tabular}

SD, standard deviation. 
of them had neurological complications with seizures (35 of 1,193,

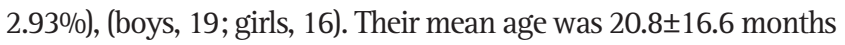
(range, 7 weeks-87 months) (Table 1). Most of them were younger than 36 months ( 32 of 35, 91.4\%). The peak incidence month was November, and the seasonal distribution of incidence corresponded with the outbreak report of Korea Centers for Disease Control and Prevention (Fig. 1).

\section{Neurologic manifestations with seizures}

The most common neurologic manifestation was febrile seizure, identified in 27 patients ( 27 of 35, 77.1\%); afebrile seizures without meningitis or encephalopathy were observed in 5 ( 5 of 35, 14.3\%); seizures with meningitis were found in 2 ( 2 of 35, 5.7\%); and seizures with encephalopathy were reported in 1 (1 of 35, 2.9\%). Eight of the 35 patients (22.9\%) did not present with respiratory symptoms such as pneumonia or bronchiolitis. In 15 patients, coinfections were identified ( 15 of 35, 42.9\%). The details of overall clinical characteristics are described in Table 1.

For 21 patients (21 of 35, 60.0\%), EEG signals were recorded, and six of them (6 of $21,28.6 \%$ ) showed abnormal focal discharges.
Neuroimages were acquired for 26 patients (26 of 35, 74.3\%), and 4 of them ( 4 of 26, 15.4\%) showed abnormal results. The CSF examinations were performed in 21 patients, and 5 of them ( 5 of $21,23.8$ \%) showed abnormal results: CSF leukocytosis in 2 patients (38.5 $\pm 37.5 / \mathrm{mm}^{3}$; range, $\left.12-65 / \mathrm{mm}^{3}\right)$ and high opening pressure ( $>18$ $\mathrm{cmH}_{2} \mathrm{O}$; range, $20-26 \mathrm{cmH}_{2} \mathrm{O}$ ) in 3 patients.

Among the patients with febrile seizure (boys, 15; girls, 12; mean age, 23.4 \pm 16.8 months), simple febrile seizure was observed in 13 (48.1\%) and complex febrile seizure in 14 (51.9\%). The mean age of patients with simple febrile seizure $(24.3 \pm 13.1$ months) and those with complex febrile seizure (22.6 \pm 20.1 months) was similar. The characteristics of complex febrile seizures included multiple seizures in a day (10 of 14, 71.4\%) and and prolonged seizures, of over 20 minutes ( 4 of 14, 28.6\%). Abnormal EEG signals were observed in 2 patients (both with complex febrile seizure): epileptiform discharges from the frontal $(n=1)$ and fronto-central $(n=1)$ areas. Brain MRI abnormalities were observed in 2 patients (both with complex febrile seizure): decreased perfusion in the left frontal lobe $(n=1)$ and benign lipoma $(n=1)$. Abnormal CSF pressure was observed in 3 of the patients: simple febrile seizure $(n=1)$ and complex febrile seizure
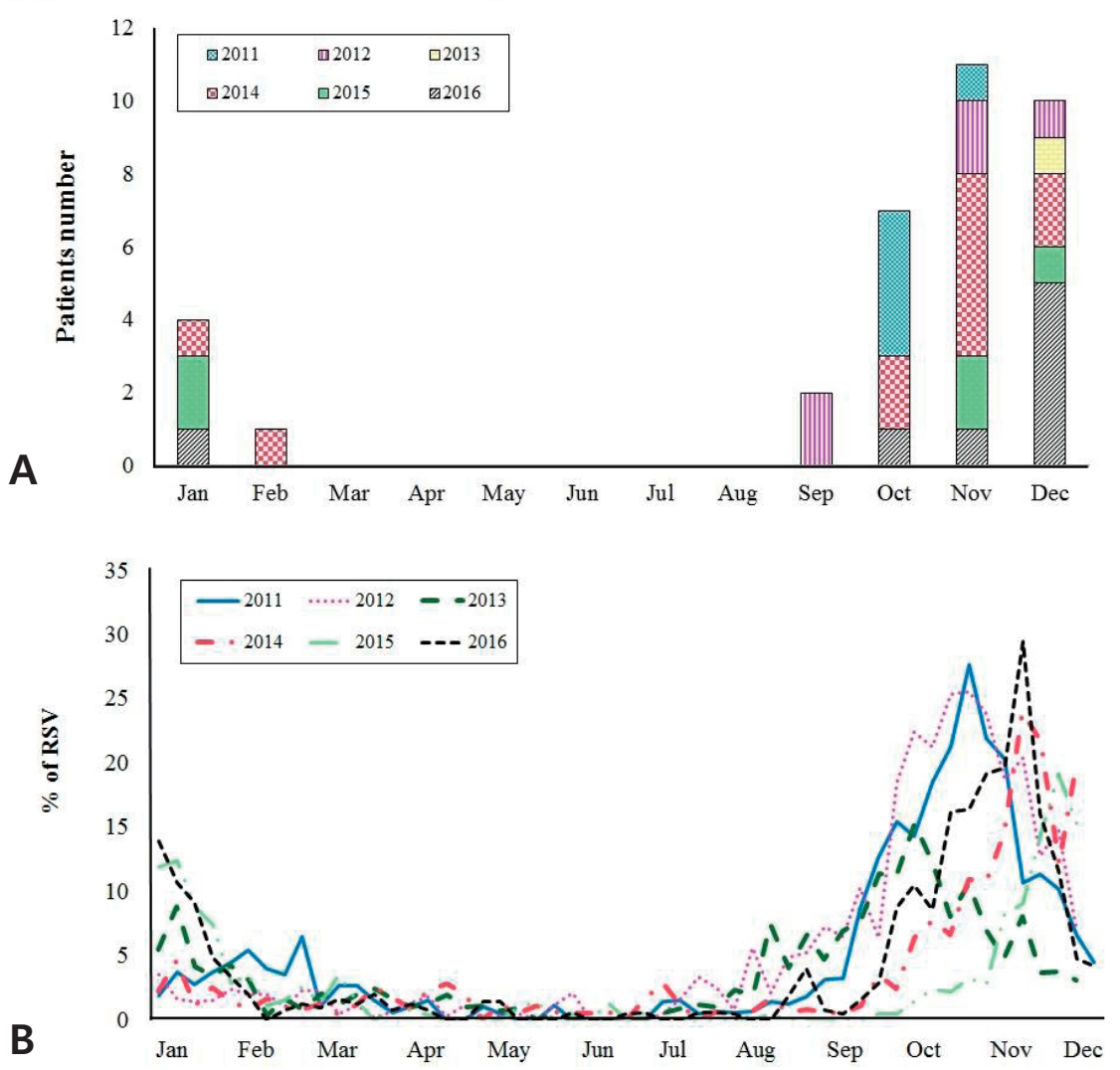

Fig. 1. (A) Seasonal distribution of patients with respiratory syncytial virus (RSV)-associated seizures in 2011-2016, showing that the peak incidence month was November. (B) Seasonal distribution of national RSV report of the Korea Centers for Disease Control and Prevention (KCDC) in 2011-2016. The distribution of the incidence of RSV-associated seizures was consistent with the report of the RSV epidemic by KCDC consistent with the report of the RSV epidemic by KCDC. 
$(n=2)$. Details of the clinical profiles of febrile seizure are described in Table 2. All the patients with febrile seizure recovered without complication.

Five patients showed afebrile seizures without meningitis or encephalopathy (boy, 4; girl, 1; mean age, 16.8 \pm 14.4 months). The types of seizure were as follows: generalized tonic-clonic $(n=2)$, generalized tonic $(n=1)$, hypomotor $(n=1)$, and myoclonic $(n=1)$. The patients had no family history of epilepsy and showed respiratory symptoms. Abnormal EEG signals were observed in 2 of the patients: focal epileptiform discharges from the parietal $(n=1)$ and occipital ( $n=1)$ areas. Brain MRI abnormalities were observed in one of the patients: benign cavernous angioma. CSF examinations were performed in 4 patients, and all of them showed normal results. One of the patients who presented with epileptiform discharges from the

Table 2. Detailed characteristics of patients with respiratory syncytial virus infection and febrile seizure

\begin{tabular}{|c|c|c|c|c|c|c|c|c|}
\hline Patient & $\begin{array}{l}\text { Sex/age } \\
\text { (mo) }\end{array}$ & Seizure type & Features & Other conditions & CSF & Brain image & EEG & Outcome \\
\hline 1 & $\mathrm{M} / / 19$ & S, GTC & - & Bronchiolitis & ND & ND & ND & Recovered \\
\hline 2 & $\mathrm{~F} / 13$ & S, GT & - & - & $\mathrm{NL}$ & CT (NL) & ND & Recovered \\
\hline 3 & $\mathrm{~F} / 18$ & S, GTC & - & Pneumonia (rhinovirus, mycoplasma) & ND & ND & ND & Recovered \\
\hline 4 & $F / 21$ & S, GT & - & Pneumonia & ND & ND & ND & Recovered \\
\hline 5 & $M / 15$ & S, GTC & - & Bronchiolitis & ND & ND & ND & Recovered \\
\hline 6 & $\mathrm{M} / 19$ & S, GTC & - & Bronchiolitis & ND & ND & ND & Recovered \\
\hline 7 & $M / 55$ & S, GTC & - & Bronchitis & ND & ND & ND & Recovered \\
\hline 8 & $F / 18$ & S, GTC & - & Pneumonia & $\mathrm{NL}$ & $\mathrm{CT}(\mathrm{NL})$ & ND & Recovered \\
\hline 9 & $\mathrm{~F} / 49$ & S, GTC & - & Pneumonia (rhinovirus) & ND & ND & ND & Recovered \\
\hline 10 & $\mathrm{~F} / 30$ & S, GTC & - & - & ND & ND & ND & Recovered \\
\hline 11 & $M / 23$ & S, GTC & - & Pneumonia (mycoplasma) & ND & ND & ND & Recovered \\
\hline 12 & $\mathrm{~F} / 14$ & S, GTC & - & - & $\mathrm{NL}$ & MRI (NL) & ND & Recovered \\
\hline 13 & $M / 21$ & S, GTC & - & - & ND & $\mathrm{CT}(\mathrm{NL})$ & ND & Recovered \\
\hline 14 & $\mathrm{~F} / 12$ & C, GTC & 3 times/day & Bronchitis & $\mathrm{NL}$ & MRI (NL) & NL & Recovered \\
\hline 15 & $F / 19$ & C, GTC & 2 times/day & Bronchiolitis & $\mathrm{NL}$ & MRI (decreased focal perfusion) & Focal spikes (fronto-central) & Recovered \\
\hline 16 & $F / 21$ & C, GTC & 2 times/day & Pneumonia (mycoplasma) & NL & $\mathrm{CT}(\mathrm{NL})$ & NL & Recovered \\
\hline 17 & $\mathrm{M} / 17$ & C, GTC & 3 times/day & Pneumonia & $\mathrm{NL}$ & $\mathrm{CT}(\mathrm{NL})$ & ND & Recovered \\
\hline 18 & $\mathrm{M} / 15$ & C, GTC & 2 times/day & - & $\mathrm{NL}$ & MRI (NL) & ND & Recovered \\
\hline 19 & $M / 31$ & C, GTC & $>20 \min$ & Pneumonia (parainflu) & $\mathrm{NL}$ & $\mathrm{MRI}(\mathrm{NL})$ & $\mathrm{NL}$ & Recovered \\
\hline 20 & $M / 4$ & C, hypomotor & $>20 \min$ & Bronchiolitis (rhinovirus, coronavirus) & $\mathrm{NL}$ & $\mathrm{MRI}(\mathrm{NL})$ & NL & Recovered \\
\hline 21 & $\mathrm{M} / 40$ & C, GTC & 2 times/day & Bronchitis & $\mathrm{NL}$ & $\mathrm{CT}(\mathrm{NL})$ & ND & Recovered \\
\hline 22 & M/87 & C, GTC & $>20 \min$ & - & $\mathrm{NL}$ & MRI (Lipoma) & $\mathrm{NL}$ & Recovered \\
\hline 23 & $\mathrm{~F} / 20$ & C, GTC & 2 times/day & Bronchitis (adenovirus) & ND & MRI (NL) & ND & Recovered \\
\hline 24 & $\mathrm{M} / 12$ & $\mathrm{C}, \mathrm{GC}$ & 2 times/day & Pneumonia & ND & $\mathrm{MRI}(\mathrm{NL})$ & $\mathrm{NL}$ & Recovered \\
\hline 25 & $F / 23$ & C, GTC & 3 times/day & Pneumonia (rhinovirus) & $\mathrm{NL}$ & CT (NL) & Diffuse slowing & Recovered \\
\hline 26 & $M / 18$ & C, GTC & 2 times/day & - & $\mathrm{NL}$ & MRI (NL) & Focal spikes (frontal) & Recovered \\
\hline 27 & $\mathrm{M} / 30$ & C, GT & $>20 \min$ & Pneumonia & ND & CT (NL) & $\mathrm{NL}$ & Recovered \\
\hline
\end{tabular}

S, simple; C, complex; GTC, generalized tonic clonic; GT, generalized tonic; GC, generalized clonic; ND, not done; NL, normal; CT, computed tomography; MRI, magnetic resonance image; EEG, electroencephalography.

Table 3. Detailed characteristics of patients with respiratory syncytial virus infection and afebrile seizure

\begin{tabular}{lclclccrc}
\hline Patient & $\begin{array}{c}\text { Sex/age } \\
\text { (mo) }\end{array}$ & $\begin{array}{c}\text { Seizure } \\
\text { type }\end{array}$ & $\begin{array}{c}\text { Previous } \\
\text { seizure }\end{array}$ & \multicolumn{1}{c}{ Other conditions } & CSF & Brain image & EEG & Outcome \\
\hline 1 & M//2 & GTC & No & Bronchiolitis & NL & MRI (NL) & Recovered \\
2 & M/35 & Hypomotor & No & Bronchitis (adenovirus) & NL & MRI (NL) & Focal spikes (left parietal) & Epilepsy \\
3 & M/22 & Myoclonic & No & Pneumonia (adenovirus, coronavirus) & ND & MRI (NL) & NL & Recovered \\
4 & F/23 & GTC & Yes & Pneumonia (rhinovirus) & NL & CT (NL) & RD \\
5 & M/2 & GC & No & Bronchiolitis & NL & MRI (cavernous angioma) & Focal spikes (parieto-occipital) Recovered \\
\hline
\end{tabular}

CSF, cerebrospinal fluid; EEG, electroencephalography; GTC, generalized tonic clonic; NL, normal; ND, not done; MRI, magnetic resonance image. 
parietal area during the first seizure later evolved to epilepsy. Further details of the clinical profiles of afebrile seizures are described in Table 3.

Seizure with meningitis, confirmed by CSF examination, was observed in two patients (both girls; mean age, $4.0 \pm 4.2$ months). One of the patients was a 7-month-old girl with urinary tract infection; she did not present with definite respiratory symptoms and showed abnormal EEG signals (continuous theta on left frontal electrode). In the patients with meningitis, CSF bacterial cultures and tests for enterovirus yielded negative results. Brain MRI results were normal in both patients. We did not perform the CSF examinations for the RSV. All the patients recovered without complication. Seizure with encephalopathy was observed in 1 patient. Detailed findings have been described below.

\section{A case with encephalopathy and transient MRI changes}

The patient was a 7-week-old female baby who visited our hospital due to respiratory difficulty, high fever $\left(38.7^{\circ} \mathrm{C}\right)$, and lethargy. Her 3-year-old elder brother had had a respiratory infection recently. Before admission, the patient was healthy and had no family history of seizure disorders. On the day of admission, she experienced three consecutive generalized tonic-clonic seizures with fever. Consciousness recovery between seizures was uncertain. After the administration of intravenous lorazepam and phenobarbital, the seizures stopped. A polymerase chain reaction-based virus test using nasopharyngeal swab revealed RSV infection; CSF cytological tests, biochemical tests, and bacterial cultures yielded no abnormalities. Brain MRI with DWI performed at first hospital day revealed high signal intensity (HSI) at the internal capsule and splenium of corpus callosum on DWI and subtle low signals on apparent diffusion coefficient (ADC) imaging at the same area. The HSI on DWI rapidly disappeared by the fourth hospital day, suggesting that the mechanism of encephalopathy was transient cytotoxic edema (Fig. 2). The EEG recordings showed sharp discharges from the frontotemporal area. The patient was admitted to the PICU, and gradually recovered over a week. In a follow-up visit at 12 months of age, neurological complication was not found and the abnormal discharges on EEG had disappeared.

\section{Discussion}

In the present study, we reported overall RSV-associated seizures in children. In summary, febrile seizure was the most common followed by afebrile seizure. Notably, the incidence of complex febrile seizure was higher, even more than that of simple febrile seizure. Although rare, RSV-associated meningitis and encephalopathy also accompanied seizures. Brain DWI of a patient with encephalopathy showed transient white matter change, which was presumed to be cytotoxic edema. The outcomes were mostly favorable, including that of the patient with encephalopathy, and one of the patients who presented with afebrile seizure evolved to epilepsy later.

An enveloped virus with negative-sense single-stranded RNA genome, RSV belongs to the Paramyxoviridae family. ${ }^{9,19}$ According to a recent systematic review, 33.1 million children aged less than 5 years are estimated to exhibit RSV-associated acute lower respiratory infections globally. Among them, 3.2 million are hospitalized and 59,600 cases result in in-hospital deaths.) Beside the common respiratory manifestations, extrapulmonary symptoms can be caused by RSV infection of the heart, liver, kidney, endocrine, and central nervous system. ${ }^{3,9)}$

Although uncommon, central nervous system involvement is an important complication of RSV infection. The representative neurological manifestations are encephalopathy, seizures, and apnea. The rare complications are strabismus, acute axonal polyneuropathy, and diaphragmatic flutter. ${ }^{4,20,21)}$ However, most of the previous studies have focused on acute encephalopathy and PICU cases, and only a few have investigated the milder febrile seizure and afebrile seizures. ${ }^{11,12,22)}$ In the present study, we included overall
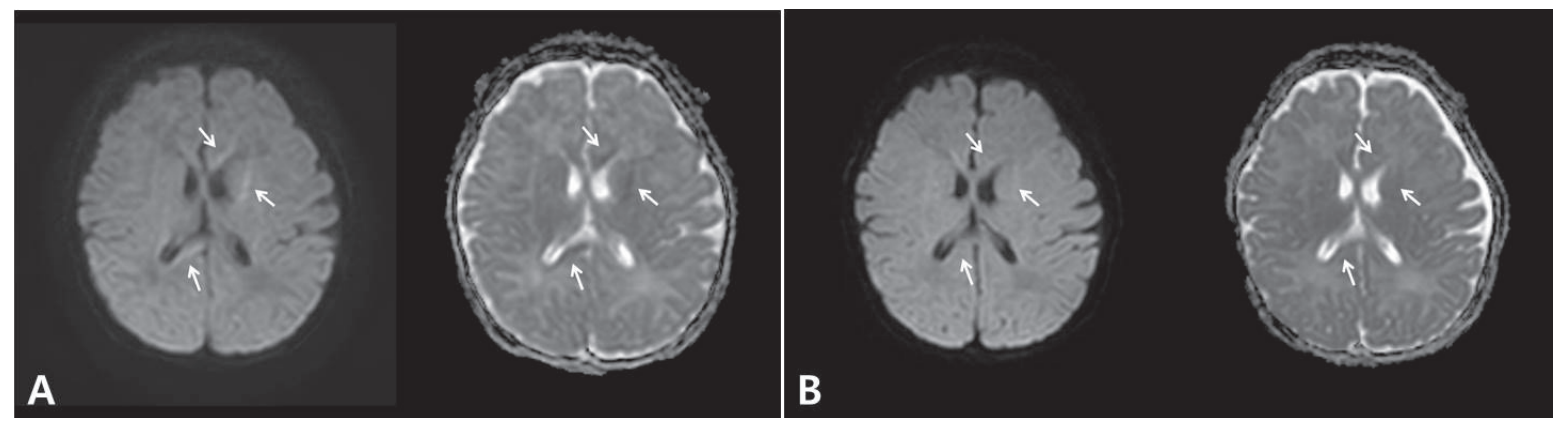

Fig. 2. (A) Brain magnetic resonance imaging (MRI) with diffusion-weighted imaging (DWI) on the first hospital day shows high signal intensity (HSI) at the internal capsule (upper and middle arrow) and splenium of the corpus callosum (lower arrow) on DWI (left) and subtle low signals on apparent diffusion coefficient (ADC) imaging (right) in the same area. (B) Brain MRI with DWI (left) and ADC (right) on the fourth hospital day shows disappearance of HSI on DWI, suggesting that the mechanism of encephalopathy was transient cytotoxic edema. 
seizures, which were reasonably presumed to be caused by RSV infection.

Febrile seizure may occur in any condition with acute elevation of body temperature in genetically predisposed children. The peak incidence age is approximately 18 months ${ }^{23)}$ Most of the febrile seizures are generalized, with approximately one-third of the patients exhibiting complex febrile seizure and febrile status epilepticus occurring in 5\% of the cases. ${ }^{24)}$ However, the RSV-related febrile seizure have not been well-characterized. The well-known study of Sweetman et al.") reported the incidence of neurological complication associated with RSV to be $1.2 \%$ (12 of 964 patients). The authors included 1 patient with complex febrile seizure but excluded five patients with simple febrile seizure. In the present study, the incidence of RSV-associated seizures, including febrile seizure, was $2.9 \%$, which is higher than the finding of Sweetman et al. ${ }^{7)}$ The global features of febrile seizure observed in this study were similar to those of the generally known febrile seizure, such as age and benign nature. However, the incidence of complex febrile seizure was higher than that of simple febrile seizure. Most of the patients showed multiple seizures in a day, which may be a distinguishing characteristic of RSV-associated febrile seizure. The reason of higher incidence of complex febrile seizure in RSVassociated febrile seizure is unclear. Previous studies reported the increased levels of CSF proinflammatory cytokines such as interleukin (IL)-6, IL-8, monocyte chemoattractant protein-1 and macrophage inflammatory protein- $1 \beta$ in patients with encephalopathy who had RSV infection. Although febrile seizure is not as severe as encephalopathy is, RSV-specific cytokine responses may be related with multiple or prolonged RSV-associated febrile seizures. ${ }^{11,25,26)}$ As RSV is one of the common infections in young children, there should be further studies of the characteristics of febrile seizure in RSV infection.

The incidence of RSV-associated afebrile seizure in Japanese children has been reported to be $0.59 \%$ ( 6 of 1,019 patients), which is similar to the incidence reported in our study $\left(0.42 \%, 5\right.$ of 1,193). $\left.{ }^{6}\right)$ The authors suggested that the RSV-associated afebrile seizures were situation-related, and prognoses were quite favorable. ${ }^{6}$ In our study population, the seizures were of various types, and in one of the cases, progressed to epilepsy. It is uncertain whether the RSV infection was merely incidental or might have influenced the occurrence of epilepsy. Further studies are needed to clarify the role of RSV in epilepsy.

Encephalopathy is a well-recognized RSV-associated complication, and its incidence has been reported to be $1.8 \%{ }^{12)} \mathrm{RSV}$ associated encephalopathies are often present with seizure. ${ }^{12)}$ Millichap and Wainwright ${ }^{5}$ reported a greater severity of the neurological complications of RSV infection. They reported the cases of 9 patients, of which $4(44 \%)$ were complicated by cardiac arrest, and 2 of the patients died. ${ }^{5)}$ Uda and Kitazawa ${ }^{8)}$ reported that the RSV-positive status epilepticus group required more ventilator care and antiepileptic drug treatment than did the RSV-negative status epilepticus group, and nearly one-third of them developed encephalopathy and profound neurological sequelae. In the present study, encephalopathy with seizure was rare and observed in only one patient. She recovered without complications, and DWI showed transient changes.

There are various RSV-associated neuroradiological findings. In most cases, brain MRI reveals no changes. However, the findings of brain edema on computed tomography and focal HSI on MRI (fluid attenuation inversion recovery and DWI) in CSF RSV-positive patients with seizures in another study suggest a pathogenic heterogeneity. ${ }^{11}$ Furthermore, DWI abnormalities in the globus pallidus in the patient suggest ischemic changes. ${ }^{5}$ In the study by Park et al., ${ }^{16)}$ the abnormal MRI findings for RSV-related encephalitis were rhombenmesencephalitis, limbic encephalitis, and acute disseminated encephalomyelitis, and diffusion abnormality on DWI was not found. In our encephalopathy case, the patient showed transient HSI on DWI on the first day of acute symptoms; this rapidly disappeared over 3 days. Our case suggests that cytotoxic edema is one of the pathogenic mechanisms underlying RSV-associated encephalopathy. Moreover, DWI can detect the early changes in encephalitis, and appropriate timing of MRI is important to reveal the dynamic changes. ${ }^{27)}$ In the very acute stage, restricted diffusion can be detected; in the subacute stage, diffusion restriction diminishes and the $\mathrm{ADC}$ value increases. ${ }^{27)} \mathrm{A}$ good outcome is usually related with the absence of cytotoxic edema, because it implies cellular edema, which may result in cellular death. ${ }^{27)}$ However, the outcome of our patient was favorable; hence, cytotoxic edema of white matter in RSV-associated encephalopathy may not always be associated with poor outcome. Transient white matter cytotoxic edema is rare, and has been previously reported in a child with traumatic brain injury, whose DWI and ADC revealed restricted diffusion in the corpus callosum and peri-atrial white matter. ${ }^{28,29)}$ The abundant $\mathrm{N}$-methyl-D-aspartate-receptors in the corpus callosum may lead to intracellular calcium entry. ${ }^{28}$ Increased $\mathrm{Ca}^{2+}$ activates enzymes such as ATPase, phospholipase, protease, and endonucleases can lead to the cell injury and death. ${ }^{30}$

The limitation of this study lies in not including the nonseizure RSV-associated neurological complications and the inability to test RSV from CSF. Considering the high incidence and morbidity of RSV infection in young children, more studies are needed in the future.

We investigated the RSV-associated neurologic complications which are presented with seizures. It should be kept in mind that during an RSV epidemic, febrile seizure is more likely to repeat within 24 hours than is generally expected. Furthermore, DWI can detect early brain changes and reflect the pathophysiology of RSVassociated encephalopathy. 


\section{Conflicts of interest}

No potential conflict of interest relevant to this article was reported.

\section{Acknowledgments}

This work was supported by the research fund of Hanyang University (HY-2016).

\section{References}

1. Shi T, McAllister DA, O’Brien KL, Simoes EAF, Madhi SA, Gessner BD, et al. Global, regional, and national disease burden estimates of acute lower respiratory infections due to respiratory syncytial virus in young children in 2015: a systematic review and modelling study. Lancet 2017;390:946-58.

2. Collins PL, Graham BS. Viral and host factors in human respiratory syncytial virus pathogenesis. J Virol 2008;82:2040-55.

3. Bohmwald K, Espinoza JA, Becerra D, Rivera K, Lay MK, Bueno SM, et al. Inflammatory damage on respiratory and nervous systems due to hRSV infection. Curr Opin Immunol 2015;36:14-21.

4. Eisenhut M. Extrapulmonary manifestations of severe respiratory syncytial virus infection--a systematic review. Crit Care 2006;10: R107.

5. Millichap JJ, Wainwright MS. Neurological complications of respiratory syncytial virus infection: case series and review of literature. J Child Neurol 2009;24:1499-503.

6. Miyama S, Goto T. Afebrile seizures associated with respiratory syncytial virus infection: a situation-related seizure disorder in early infancy. Pediatr Int 2011;53:113-5.

7. Sweetman LL, Ng YT, Butler IJ, Bodensteiner JB. Neurologic complications associated with respiratory syncytial virus. Pediatr Neurol 2005;32:307-10.

8. Uda K, Kitazawa K. Febrile status epilepticus due to respiratory syncytial virus infection. Pediatr Int 2017;59:878-84.

9. Bohmwald K, Espinoza JA, Rey-Jurado E, Gómez RS, González PA, Bueno SM, et al. Human respiratory syncytial virus: infection and pathology. Semin Respir Crit Care Med 2016;37:522-37.

10. Moon JH, Na JY, Kim JH, Yum MK, Oh JW, Kim CR, et al. Neurological and muscular manifestations associated with influenza B infection in children. Pediatr Neurol 2013;49:97-101.

11. Kawashima H, Ioi H, Ushio M, Yamanaka G, Matsumoto S, Nakayama T. Cerebrospinal fluid analysis in children with seizures from respiratory syncytial virus infection. Scand J Infect Dis 2009;41:228-31.

12. Ng YT, Cox C, Atkins J, Butler IJ. Encephalopathy associated with respiratory syncytial virus bronchiolitis. J Child Neurol 2001;16:1058.
13. Kho N, Kerrigan JF, Tong T, Browne R, Knilans J. Respiratory syncytial virus infection and neurologic abnormalities: retrospective cohort study. J Child Neurol 2004;19:859-64.

14. Chung B, Wong V. Relationship between five common viruses and febrile seizure in children. Arch Dis Child 2007;92:589-93.

15. Yoon SW, Kim HJ, Yum MK, Seol IJ. Neurologic complications associated with respiratory syncytial virus infection in childhood. J Korean Child Neurol Soc 2008;16:206-12.

16. Park A, Suh SI, Son GR, Lee YH, Seo HS, Eun BL, et al. Respiratory syncytial virus-related encephalitis: magnetic resonance imaging findings with diffusion-weighted study. Neuroradiology 2014;56: 163-8.

17. Park A, Suh S. Respiratory syncytial virus-related encephalitis: magnetic resonance imaging findings with diffusion-weighted study: response to a "letter to the editor". Neuroradiology 2014;56:433.

18. Capovilla G, Mastrangelo M, Romeo A, Vigevano F. Recommendations for the management of "febrile seizures": Ad Hoc Task Force of LICE Guidelines Commission. Epilepsia 2009;50 Suppl 1:2-6.

19. Hacking D, Hull J. Respiratory syncytial virus--viral biology and the host response. J Infect 2002;45:18-24.

20. Adams JA, Zabaleta IA, Sackner MA. Diaphragmatic flutter in three babies with bronchopulmonary dysplasia and respiratory syncytial virus bronchiolitis. Pediatr Pulmonol 1995;19:312-6.

21. Eisenhut M. Extrapulmonary manifestations of severe RSV bronchiolitis. Lancet 2006;368:988.

22. Morichi S, Kawashima H, Ioi H, Yamanaka G, Kashiwagi Y, Hoshika A, et al. Classification of acute encephalopathy in respiratory syncytial virus infection. J Infect Chemother 2011;17:776-81.

23. Berg AT, Shinnar S. Complex febrile seizures. Epilepsia 1996;37:12633.

24. Patel N, Ram D, Swiderska N, Mewasingh LD, Newton RW, Offringa M. Febrile seizures. BMJ 2015;351:h4240.

25. Kawashima H, Kashiwagi Y, Ioi H, Morichi S, Oana S, Yamanaka G, et al. Production of chemokines in respiratory syncytial virus infection with central nervous system manifestations. J Infect Chemother 2012;18:827-31.

26. Zlateva KT, Van Ranst M. Detection of subgroup B respiratory syncytial virus in the cerebrospinal fluid of a patient with respiratory syncytial virus pneumonia. Pediatr Infect Dis J 2004;23:1065-6.

27. Donmez FY. Respiratory syncytial virus-related encephalitis: magnetic resonance imaging findings with diffusion-weighted study. Neuroradiology 2014;56:431-2.

28. Al Brashdi YH, Albayram MS. Reversible restricted-diffusion lesion representing transient intramyelinic cytotoxic edema in a patient with traumatic brain injury. Neuroradiol J 2015;28:409-12.

29. Takayama H, Kobayashi M, Sugishita M, Mihara B. Diffusion-weighted imaging demonstrates transient cytotoxic edema involving the corpus callosum in a patient with diffuse brain injury. Clin Neurol Neurosurg 2000;102:135-9.

30. Kovac S, Domijan AM, Walker MC, Abramov AY. Seizure activity results in calcium- and mitochondria-independent ROS production via NADPH and xanthine oxidase activation. Cell Death Dis 2014;5: e1442. 